\title{
Optic Nerve Sonography in a Patient with Osteopetrosis
}

\author{
Raffaele Nardone, Francesco Brigo, Andrea Orioli, Frediano Tezzon, Eugen Trinka, \\ Piergiorgio Lochner
}

Can J Neurol Sci. 2014; 41: 400-401

Osteopetrosis produces abnormally dense and brittle bone that is prone to fracture. ${ }^{1}$ Cranial nerve dysfunction often occurs as the result of stenosis of foramina at the skull base. The cause of visual disturbances in osteopetrosis may be due to a compressive optic neuropathy from narrowing at the optic canal or may be due to increased intracranial pressure.

Optic nerve sonography (US) has been used to identify and monitor intracranial hypertension through the measurement of the optic nerve sheath diameter (ONSD), since increased intracranial pressure of different aetiologies leads to enlargement of the ONSD. ${ }^{2,3}$

We describe a patient with visual disturbances from osteopetrosis-related optic nerve edema due to enhanced intracranial pressure.

A 22-year-woman with osteopetrosis was referred to our hospital complaining of visual disturbances. In particular she noted, since two weeks prior, transient bilateral visual obscurations. The patient was taking the contraceptive pill and was overwight (her body mass index was 28). On examination, visual acuity was 7/10. Pupil, motility, intraocular pressure, and slit lamp examinations were normal. Computerized visual fields analysis showed bilateral blind spot enlargement. There was grade 2 to 3 bilateral optic nerve edema (according to the Modified Frisén Scale $)^{4}$ with superimposed mild pallor. Magnetic resonance imaging and computed tomography scans showed thickening of the skull, especially at the skull base. A magnetic resonance venography showed no evidence of sinus thrombosis. The lumbar puncture revealed an increased opening pressure of $35 \mathrm{~cm} \mathrm{H}_{2} 0$, but otherwise normal cerebrospinal fluid (cell count, glucose and protein levels). Ultrasound examinations of the eye were carried out with the same procedure previously described. ${ }^{2,3}$ Transorbital US was carried out in B-mode using a Vivid Seven ultrasound system and a 7-11 $\mathrm{MHz}$ linear array transducer (General Electric Healthcare, Milwaukee, WI, USA). The patient was examined in supine position with the upper part of the body and the head elevated to $20-30^{\circ}$. For safety reasons of biomechanical side-effects, the mechanical index (MI) was reduced to 0.2 . The probe was placed on the temporal part of the closed upper eyelid using a thick layer of ultrasound gel. The anterior part of the optic nerve was depicted in an axial plane showing the papilla and the optic nerve in its longitudinal course. ONSD was measured $3 \mathrm{~mm}$ behind the globe. ${ }^{4}$ In order to gauge the ONSD, the distance between the external borders of the hyperechogenic area surrounding the optic nerve was quantified. Each bulb was examined three times and means were calculated.



Figure 1: Axial computed tomography scan of the patient with osteopetrosis. Thickening of the bones at the skull base was observed but the optic canals do not appear significantly stenotic.

From the Department of Neurology (RN, FB, AO, FT, PL), Franz Tappeiner Hospital, Meran/o; Department of Neurological, Neuropsychological, Morphological and Movement Sciences, Section of Clinical Neurology (FB), University of Verona; Department of Neurology (PL), University Amedeo Avogadro, Novara, Italy; Department of Neurology (RN, ET), Christian Doppler Clinic, Paracelsus Medical University, Salzburg, Austria.

Received SePtember 9, 2013. Final Revisions Submitted November 5, 2013. Correspondence to: Raffaele Nardone, Department of Neurology - "F. Tappeiner" Hospital - Meran/o, Via Rossini, 5, 39012 Meran/o (BZ) - Italy.

Email: raffaele.nardone@asbmeran-o.it. 


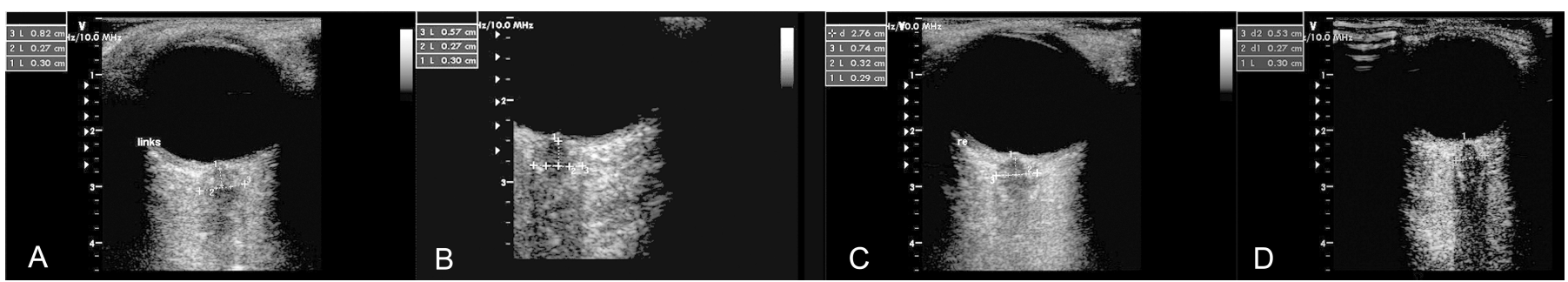

Figure 2: Optic nerve sonography before and after treatment with acetazolamide in the right $(A-B)$ and in left eye $(C-D)$. A markedly increased optic nerve sheath diameter has been detected bilaterally at the first examination. Values within the normal range have been observed after therapy.

Values obtained with the above described procedure were compared with normal values previously published..$^{2,3}$

At the first examination, optic nerve US showed markedly abnormal ONSD values (left $0.82 \mathrm{~cm}$., right $0,74 \mathrm{~cm}$.). The patient was treated with oral acetazolamide (1000 mg x 2), moreover a low-sodium weight reduction program was recommended.

At the follow-up three months after the first evaluation, only a slight and non-significant reduction in weight $(2.4 \mathrm{~kg})$ was achieved. The patient had persistent but slightly reduced papilledema, while her visual disturbances, in particular the residual field deficits had completely resolved. At that time, optic nerve US showed ONSD values within the normal range (left $0.57 \mathrm{~cm}$. right $0.53 \mathrm{~cm}$.). We described here an additional case of intracranial hypertension (IH) in osteopetrosis and first explored the utility of optic nerve US in monitoring the consequent optic neuropathy. In subjects with osteopetrosis, optic nerve edema and visual disturbances can be attributed to optic nerve compression at the optic canal because of increased intracranial pressure. The prominent base skull and calvarial thickening with limitation of intracranial volume in osteopetrosis may account for the increased intracranial pressure. Other causes include primary dysmyelination of the optic nerve, compression of the retinal vein and retinal degeneration. The determination of the precise cause for visual loss in osteopetrosis is important. In fact, optic nerve compression in the canal may respond to optic canal decompression, ${ }^{5}$ whereas optic nerve edema was found to respond to optic nerve sheath fenestration (ONSF) in a patient with osteopetrosis. ${ }^{6}$

The optic canals do not appear significantly stenotic in our patient and thus visual disturbances cannot be ascribed to optic nerve compression secondary to the narrowing of the optic foramina. Therefore, the cause of visual deterioration in our patient may be the increased intracranial pressure due to cerebral venous outflow obstruction or restriction at the foramen magnum. ${ }^{7}$

This case report illustrates the importance of early discovery of increased intracranial pressure in a patient with osteopetrosis. Before the occurrence of an irreversible visual loss due to degeneration/atrophy of the optic nerve, conventional medical therapy with acetazolamide can be successful performed. Early pharmacological treatment for this condition can make surgical procedures (such as ONSF) unnecessary.

Optic nerve US is a promising technique that is known to represent an additional diagnostic tool in the diagnostic work-up and in the follow-up of idiopathic IH. Here we extend the previous findings and demonstrate that US may also be useful in monitoring the clinical evolution and treatment efficacy in patients with IH due to osteopetrosis.

Moreover, this case report study suggests that individuals with visual disturbances and optic disk edema associated with osteopetrosis may benefit from conventional pharmacological therapy if the optic canals appear to be open and the optic nerve edema is clearly related to increased intracranial pressure.

\section{REFERENCES}

1. Tolar J, Teitelbaum SL, Orchard PJ. Osteopetrosis. N Engl J Med. 2004;351(27): 2839-49.

2. Geeraerts T, Merceron S, Benhamou D, Vigué B, Duranteau J. Noninvasive assessment of intracranial pressure using ocular sonography in neurocritical care patients. Intensive Care Med. 2008;12(Suppl 2):117.

3. Zeiler FA, Unger B, Kramer AH, Kirkpatrick AW, Gillman LM. A unique model for ultrasound assessment of optic nerve sheath diameter. Can J Neurol Sci. 2013;40(2):225-9.

4. Scott CJ, Kardon RH, Lee AG, Frisén L, Wall M. Diagnosis and grading of papilledema in patients with raised intracranial pressure using optical coherence tomography vs clinical expert assessment using a clinical staging scale. Arch Ophtalmol. 2010; 128(6):705-11.

5. Vanier V, Miller NR, Carson BS. Bilateral visual improvement after unilateral optic canal decompression vault expansion in a patient with osteopetrosis, narrowed optic canals, and increased intracranial pressure. J Neurol Neurosurg Psychiatry. 2000;69 (3):405-6

6. Allen RC, Nerad JA, Kattah JC, Lee AG. Resolution of optic nerve edema and improved visual function after optic nerve sheath fenestration in a patient with osteopetrosis. Am J Ophthalmol. 2006;141(5):945-7.

7. Hoyt CS, Billson FA. Visual loss in osteopetrosis. Am J Dis Child. 1979;133:955-8. 